\title{
miR-140-5p regulates angiogenesis following ischemic stroke by targeting VEGFA
}

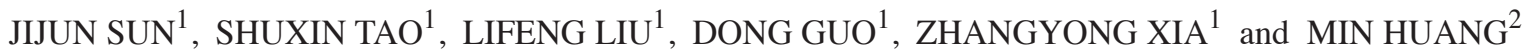 \\ ${ }^{1}$ Department of Neurology, Liaocheng People's Hospital and Liaocheng Clinical School of Taishan Medical University, \\ Liaocheng, Shandong 252000; ${ }^{2}$ Department of Neurology, Jinan University, Guangzhou, Guangdong 510632, P.R. China
}

Received May 5, 2015; Accepted February 22, 2016

DOI: $10.3892 / \mathrm{mmr} .2016 .5066$

\begin{abstract}
MicroRNA (miRNA or miR) expression profiles are altered in tissues under hypoxic-ischemic conditions. The expression of miR-140 is downregulated $>2$-fold following hypoxic-ischemic brain damage, however, its role in angiogenesis subsequent to cerebral ischemia is not fully understood. The present study aimed to investigate the role of miR-140-5p in angiogenesis and the molecular mechanism mediated by vascular endothelial growth factor A (VEGFA) in an in vitro model for brain ischemia. A rat middle cerebral artery occlusion (MCAO) model was constructed, and the results from reverse transcription-quantitative polymerase chain reaction and western blot analysis demonstrated that the expression levels of miR-140-5p were significantly decreased, while the expression levels of VEGFA were significantly increased between 12 and $48 \mathrm{~h}$ in the rat cerebral following MCAO. Furthermore, human umbilical vein endothelial cells (HUVECs) were exposed to low oxygen conditions and it was demonstrated that hypoxia downregulated miR-140-5p and upregulated VEGFA expression levels. The miR-140-5p mimic was transfected into the normoxic and hypoxic HUVECs and 3-(4,5-dimethylthiazol-2-yl)-2,5-diphenyltetrazolium bromide, Transwell migration and tube formation assays were performed. The results indicated that miR-140-5p inhibited angiogenesis by decreasing cell proliferation, migration and tube formation. Additionally, in human embryonic kidney 293 cells, results from the luciferase reporter assay revealed that miR-140-5p directly targeted the 3' untranslated region of VEGFA and that miR-140-5p regulated the protein expression of VEGFA. To further analyze this effect, a VEGFA-pEGFP-C1 plasmid was transfected into the normoxic and hypoxic HUVECs, and it was revealed that the
\end{abstract}

Correspondence to: Dr Jijun Sun, Department of Neurology, Liaocheng People's Hospital and Liaocheng Clinical School of Taishan Medical University, 67 West Dongchang Road, Liaocheng, Shandong 252000, P.R. China

Email: sun2003404@yeah.net

Key words: miR-140-5p, angiogenesis, ischemic stroke, hypoxia, vascular endothelial growth factor A inhibitory effect of miR-140-5p on angiogenesis was attenuated by the overexpression of VEGFA. In conclusion, to the best of our knowledge, the present study is the first to suggest that miR-140-5p exerts an inhibitory effect on angiogenesis in an in vitro model of ischemia, and this effect is achieved partially by targeting VEGFA. The present study provided a novel biomarker for the treatment of cerebral ischemia.

\section{Introduction}

Stroke is the predominant cause of mortality and a long-term disability worldwide (1). Ischemic stroke is more common than hemorrhagic stroke and accounts for $>80 \%$ of all stroke cases (2). Angiogenesis is associated with neurological functional recovery and it has been demonstrated to be critical in improving brain recovery following ischemic insults $(3,4)$.

Increasing evidence demonstrated that microRNAs (miRNAs or miRs) are involved in stroke (5). Peng et al (6) demonstrated that in the pathogenesis of hypoxic-ischemic brain damage, numerous miRNAs were downregulated, in particular miR-140 was downregulated $>2$ fold. miR-140 has been investigated extensively in cancer research (7-11), however, its role in angiogenesis following ischemic stroke remains unclear.

In the present study, the expression of miR-140-5p and vascular endothelial growth factor A (VEGFA) was examined in a rat model of middle cerebral artery occlusion (MCAO). Furthermore, the present study demonstrated the role of miR-140-5p in angiogenesis and the molecular mechanism mediated by VEGFA in an in vitro model for brain ischemia.

\section{Materials and methods}

Permanent rat model of MCAO. The present study was approved by the Ethics Committee of Liaocheng People's Hospital (Shandong, China). All animal experiments were performed in compliance with the guidelines for the Care and Use of Laboratory Animals published by the National Institutes of Health (12). Sprague-Dawley male rats $(n=32)$, weighing 250-300 g, aged 6-8 weeks old were purchased from SLAC Laboratory Animal, Inc. (Shanghai, China). Rats were housed in individual cages with of a 12-h light/dark cycle and a controlled temperature $\left(22-24^{\circ} \mathrm{C}\right)$, with free access to food and fresh water. The rats were sacrificed at 12, 24 and 
$48 \mathrm{~h}$ following treatments via an intraperitoneal injection of pentobarbital (200 mg/kg; Sigma-Aldrich, St. Louis, MO, USA). Each rat was anesthetized with $10 \%$ chloral hydrate (4 ml/kg; Shuanghe, Ltd., Beijing, China) intraperitoneally. Following the exposure of the right common carotid artery, a 4-0 nylon monofilament coated with a silicone tip was inserted into the internal carotid artery until mild resistance was felt, to occlude the middle cerebral artery. Rats in the MCAO group $(n=24)$ were divided into three subgroups based on the duration of ischemia for 12, 24 and $48 \mathrm{~h}$. Rats in the sham group $(n=8)$ underwent anesthesia and surgery without the occlusion of the middle cerebral artery. Following MCAO, neurological function was tested, and the score was evaluated as previously described (13). Rats with scores of 1-3 were included.

Cell culture. Human umbilical vein endothelial cells (HUVECs) and human embryonic kidney (HEK)293 cells were purchased from American Type Culture Collection (Manassas, VA, USA). HEK293 cells and HUVECs in normoxic conditions were cultured in Dulbecco's modified Eagle's medium (DMEM; Gibco; Thermo Fisher Scientific, Inc., Waltham, MA, USA) with $10 \%$ fetal bovine serum (FBS; Thermo Fisher Scientific, Inc.) at $37^{\circ} \mathrm{C}$, in a humidified atmosphere of $95 \%$ air and $5 \% \mathrm{CO}_{2}$. HUVECs in hypoxic conditions were cultured in DMEM with FBS at $37^{\circ} \mathrm{C}$, in a humidified atmosphere of $94 \% \mathrm{~N}_{2}, 5 \% \mathrm{CO}_{2}$ and $1 \% \mathrm{O}_{2}$.

Cell transfection. The miRNA control, miR-140-5p mimic and miR-140-5p inhibitor were synthesized by Bioneer (Shanghai, China). The pEGFP-C1 plasmid was purchased from Clontech Laboratories, Inc. (Mountain View, CA, USA). Transfection of miRNA control, miR-140-5p mimic, miR-140-5p inhibitor, pEGFP-C1 plasmid and VEGFA-pEGFP-C1 was performed in HUVECs and/or HEK293 cells using Lipofectamine 2000 (Invitrogen; Thermo Fisher Scientific, Inc.), according to the manufacturer's protocol. The oligonucleotides and plasmids were transfected at a concentration of 20 and $50 \mathrm{nM}$, respectively. Briefly, a day prior to transfection, the cells were seeded into 6-well plates. Lipofectamine $(10 \mu \mathrm{l})$ and oligonucleotides/plasmids were mixed in $250 \mu$ l Opti-MEM (Invitrogen; Thermo Fisher Scientific, Inc.) and incubated at room temperature for $20 \mathrm{~min}$ to form a complex. The cells were incubated with the complex at $37^{\circ} \mathrm{C}$ for $6 \mathrm{~h}$, and the medium was subsequently replaced with fresh medium.

Luciferase reporter assay. The wild type VEGFA-3' untranslated region (UTR) and mutant VEGFA-3'UTR were cloned into the psiCHECK-2 vector (Promega Corporation, Madison, WI, USA). A luciferase reporter assay was performed in HEK293 cells. The miRNA control, miR-140-5p mimic and psiCHECK-2-UTR vectors were transfected into the cells using Lipofectamine, as described above. The cells were lysed $24 \mathrm{~h}$ following the transfection and the luciferase activity was determined by the Dual-Luciferase Reporter Assay system (Promega Corporation), according to the manufacturer's protocol.

3-(4,5-dimethylthiazol-2-yl)-2,5-diphenyltetrazolium bromide (MTT) assay. The cells were plated into 96-well plates and allowed to grow for 24,48 and $72 \mathrm{~h}$ prior to incubation with $10 \mu \mathrm{l}$ MTT solution $(0.5 \mathrm{mg} / \mathrm{ml}$; Beyotime Institute of Biotechnology, Shanghai, China) for $4 \mathrm{~h}$ at $37^{\circ} \mathrm{C}$. The formazan crystals were solubilized in dimethyl sulfoxide (Sigma-Aldrich) and the optical density was measured at $570 \mathrm{~nm}$ using a microplate reader (SpectraMax M2e; Molecular Devices, LLC, Sunnyvale, CA, USA).

Transwell migration assay. A Transwell migration assay was performed using a 6-well Transwell plate with $8.0 \mu \mathrm{m}$ pore polycarbonate membrane inserts (Corning, Inc., Corning, NY, USA). The HUVECs were suspended and seeded at a density of $5 \times 10^{4}$ cells $/ \mathrm{ml}$ into the upper chambers with serum-free medium. Culture medium with $10 \%$ FBS was added to the lower chambers. Following $24 \mathrm{~h}$ incubation, the cells that migrated were fixed in $95 \%$ ethanol and stained with hematoxylin solution (Sigma-Aldrich) for $15 \mathrm{~min}$ at room temperature. The cells were then counted under a TS100 inverted microscope (Nikon Corporation, Tokyo, Japan).

In vitro tube formation assay. Pre-cooled Matrigel (BD Biosciences, Franklin Lakes, NJ, USA) was added to the 24-well plates and allowed to solidify at $37^{\circ} \mathrm{C}$ for $30 \mathrm{~min}$. The cells were subsequently suspended and seeded at a density of $4 \times 10^{5}$ cells $/ \mathrm{ml}$ into the 24 -well plates. Following incubation at $37^{\circ} \mathrm{C}$, in $5 \%$ $\mathrm{CO}_{2}$ for $18 \mathrm{~h}$, the formation of tubes was observed under the microscope and images were captured (Olympus Corporation, Tokyo, Japan). The tube lengths were analyzed using Image-Pro Plus software, version 5.0 (BD Biosciences) in five randomly selected fields per well.

Reverse transcription-quantitative polymerase chain reaction $(R T-q P C R)$. The total RNA from the ipsilateral ischemic cerebral cortex and HUVECs was extracted using TRIzol reagent (Invitrogen; Thermo Fisher Scientific, Inc.). For miRNA isolation, the mirVana miRNA Isolation kit (Ambion; Thermo Fisher Scientific, Inc.) was used, according to the manufacturer's protocol. The total RNA (1 $\mu \mathrm{g})$ was reverse-transcribed into cDNA using the Transcriptor First Strand cDNA Synthesis kit (Roche Diagnostics, Basel, Switzerland). The primer sequences used were as follows: miR-140-5p, forward: 5'-ACACTCCAG CTGGGCAGTGGTTTTACCCTA-3' and reverse: 5'-TGG TGTCGTGGAGTCG-3'; U6, forward: 5'-CTCGCTTCGGCA GCACA-3' and reverse: 5'-AACGCTTCACGAATTTGCGT-3'. PCR was performed using a SYBR Green PCR kit (Applied Biosystems; Thermo Fisher Scientific, Inc.) in a 7300 Sequence Detection system (Applied Biosystems; Thermo Fisher Scientific, Inc.), with an initial denaturation step at $95^{\circ} \mathrm{C}$ for $5 \mathrm{~min}$, followed by 40 cycles of amplification $\left(95^{\circ} \mathrm{C}\right.$ for $30 \mathrm{sec}, 60^{\circ} \mathrm{C}$ for $30 \mathrm{sec}$ and $72^{\circ} \mathrm{C}$ for $40 \mathrm{sec}$ ) and a final extension step at $72^{\circ} \mathrm{C}$ for $10 \mathrm{~min}$. The relative $\mathrm{mRNA}$ expression of $\mathrm{miR}-140-5 \mathrm{p}$ was calculated using the $2^{-\Delta \Delta \mathrm{Ct}}$ method and normalized to the U6 expression (14).

Western blot analysis. The total protein was extracted from the ipsilateral ischemic cerebral cortex and cultured cells using ice-cold lysis buffer $(50 \mathrm{mM}$ Tris- $\mathrm{HCl}, 150 \mathrm{mM} \mathrm{NaCl}, 0.1 \%$ SDS, $1 \%$ Triton X-100, 2 mM EDTA and protease inhibitor cocktail). Following centrifugation at $10,000 \mathrm{x}$ for $15 \mathrm{~min}$ at $4^{\circ} \mathrm{C}$, the supernatant was collected and 

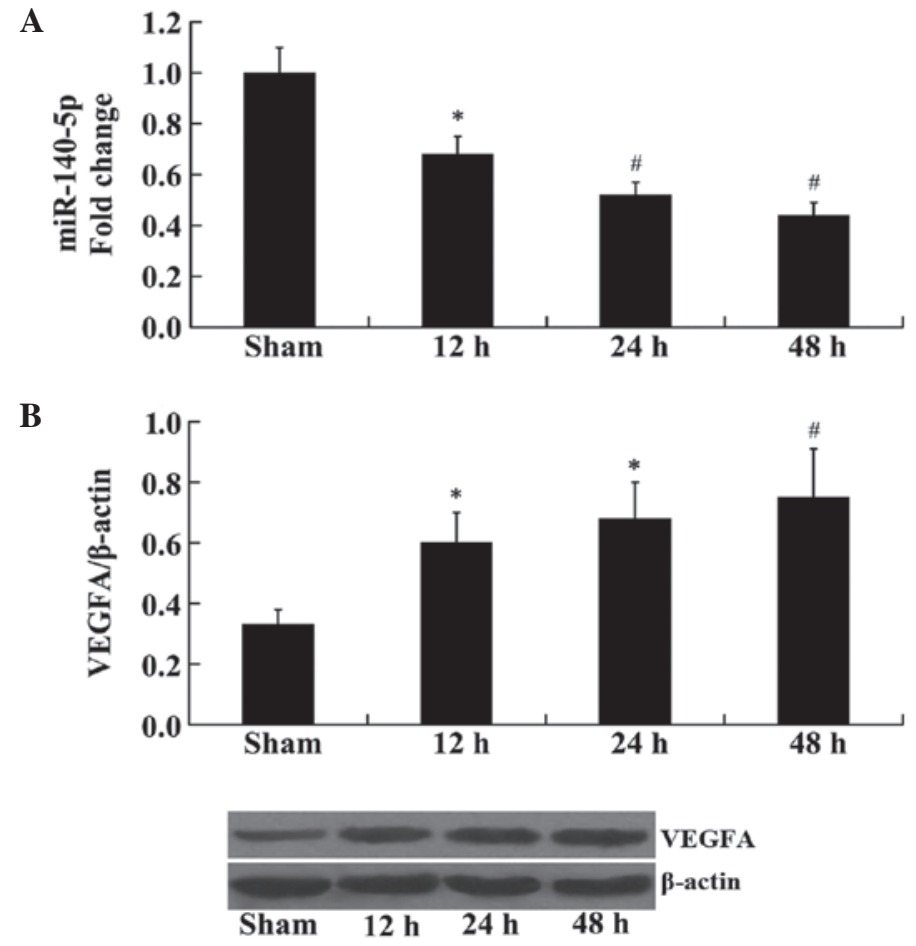

Figure 1. miR-140-5p fold change and the protein expression of VEGFA in the cerebral tissue following middle cerebral artery occlusion for $12-48 \mathrm{~h}$. (A) The fold change of miR-140-5p was normalized against the sham group. (B) The protein expression of VEGFA was normalized against $\beta$-actin. The data are expressed as the mean \pm standard deviation $\left({ }^{*} \mathrm{P}<0.05 ;{ }^{*} \mathrm{P}<0.01\right.$, vs. the sham group). VEGFA, vascular endothelial growth factor $\mathrm{A}$; miR, microRNA.

the protein concentration was determined using the Pierce BCA Protein Assay kit (Thermo Fisher Scientific, Inc.). A total of $20 \mu \mathrm{g}$ protein was separated by $12 \%$ SDS-PAGE and transferred onto polyvinylidene fluoride membranes (EMD Millipore, Billerica, MA, USA). The membranes were blocked with 5\% non-fat milk in Tris-buffered saline, containing $0.01 \%$ Tween-20 at $4^{\circ} \mathrm{C}$ overnight. Following blocking, the membranes were incubated at $37^{\circ} \mathrm{C}$ for $2 \mathrm{~h}$ with VEGFA (dilution: 1:800; cat. no: sc-53462, mouse monoclonal; Santa Cruz Biotechnology, Inc., Santa Cruz, CA, USA) and $\beta$-actin (dilution: 1:1,000; cat. no: sc-47778, mouse monoclonal; Santa Cruz Biotechnology, Inc.) primary antibodies. Following washing with phosphate-buffered saline, the membranes were incubated with the corresponding horseradish peroxidase-conjugated secondary antibody (dilution: 1:2,000; cat. no: BA1051, goat polyclonal; Wuhan Boster Biological Technology, Ltd., Wuhan, China). The reaction was detected using the Pierce ECL Western Blotting Substrate (Thermo Fisher Scientific, Inc.). The band intensity was quantified using Image J $1.48 \mathrm{u}$ software (National Institutes of Health, Bethesda, MD, USA).

Statistical analysis. Statistical analysis was performed using SPSS software, version 19.0 (IBM SPSS, Chicago, IL, USA). The data are presented as the mean \pm standard deviation. Student's t-test was used to identify differences between the two groups. $\mathrm{P}<0.05$ was considered to indicate a statistically significant difference.

\section{Results}

Expression of miR-140-5p and VEGFA in rat cerebral tissue following MCAO. RT-qPCR and western blot analyses were
A

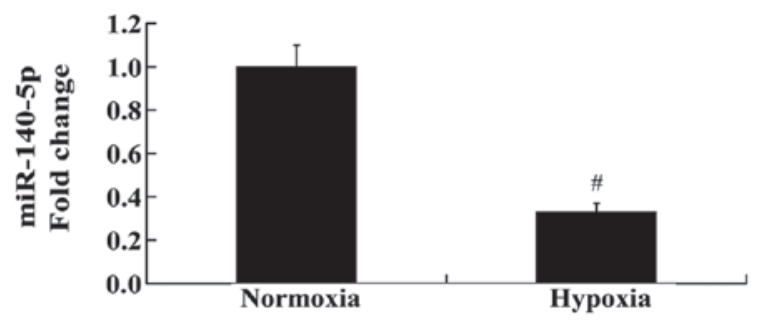

B

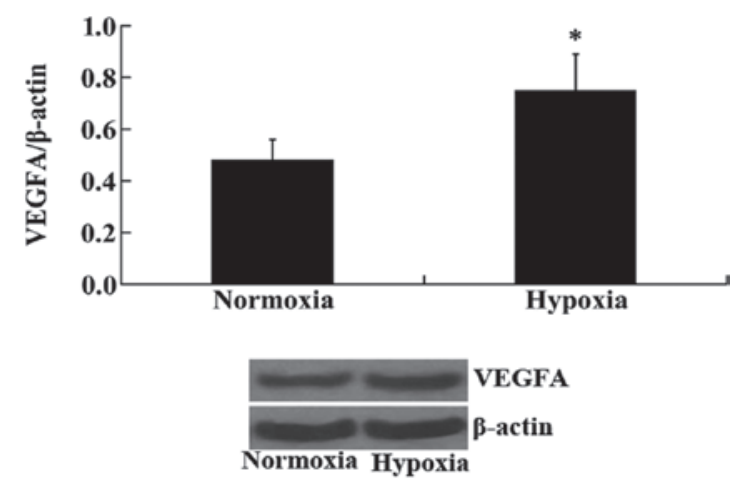

Figure 2. miR-140-5p fold change and the protein expression of VEGFA in human umbilical vein endothelial cells following hypoxia. (A) The fold change of miR-140-5p was normalized against that of the normoxia group. (B) The protein expression of VEGFA was normalized against $\beta$-actin. The data are expressed as the mean \pm standard deviation $\left({ }^{*} \mathrm{P}<0.05 ;{ }^{\#} \mathrm{P}<0.01\right.$, vs. the normoxia group) VEGFA, vascular endothelial growth factor A; miR, microRNA.

used to determine changes in the expression of miR-140-5p and VEGFA in the rat brain following MCAO at 12, 24 and $48 \mathrm{~h}$. The results from RT-qPCR analysis demonstrated that the expression of miR-140-5p was significantly reduced 
A

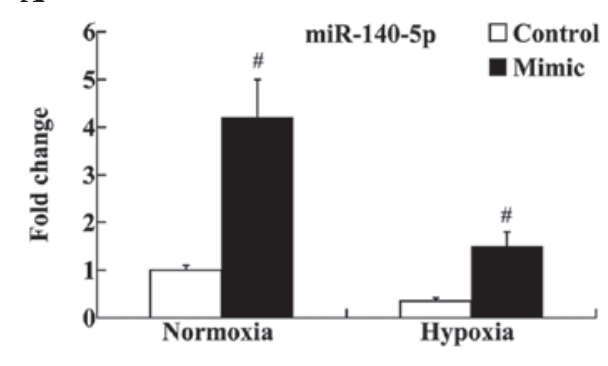

C

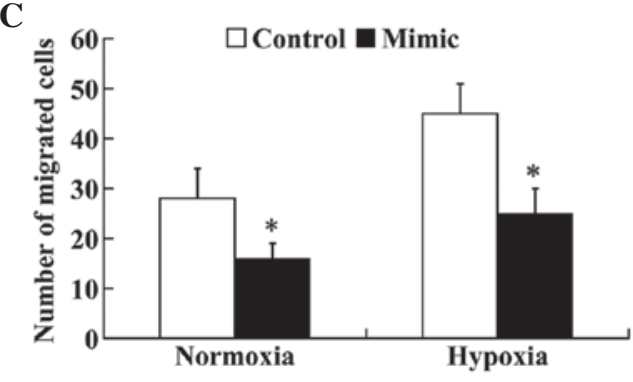

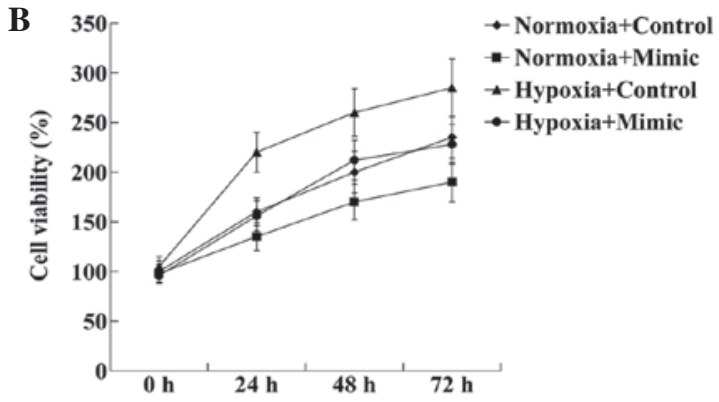

$\mathbf{D}$

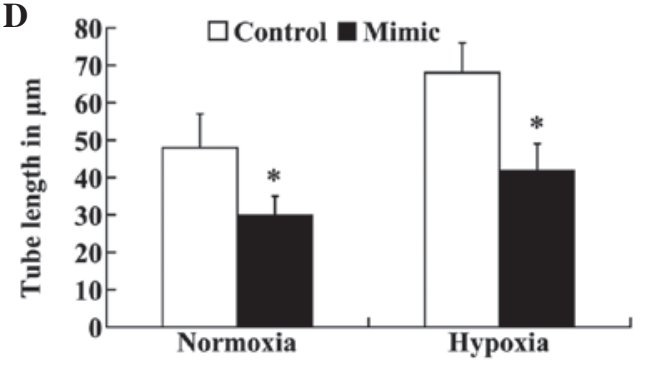

Figure 3. Effect of miR-140-5p on in vitro angiogenesis in human umbilical vein endothelial cells following transfection with the miR-140-5p mimic. (A) Fold changes of miR-140-5p was normalized against the normoxia/control group. (B) Cell viability was determined by 3-(4,5-dimethylthiazol-2-yl)-2,5-diphenyltetrazolium bromide assay. (C) The number of migrated cells was determined using a Transwell migration assay. (D) The tube length was determined using a tube formation assay. The data are expressed as the mean \pm standard deviation $\left({ }^{*} \mathrm{P}<0.05\right.$; ${ }^{~} \mathrm{P}<0.01$, vs. the sham group). miR, microRNA.

at 12, 24 and $48 \mathrm{~h}$ following MCAO, compared with the sham group $(\mathrm{P}<0.05, \mathrm{P}<0.01$ and $\mathrm{P}<0.01$, respectively; Fig. $1 \mathrm{~A})$. However, western blot analysis demonstrated that the protein expression levels of VEGFA were significantly increased at 12, 24 and $48 \mathrm{~h}$ following MCAO, compared with the sham group $(\mathrm{P}<0.05, \mathrm{P}<0.05$ and $\mathrm{P}<0.01$, respectively; Fig. $1 \mathrm{~B})$.

Expression of miR-140-5p and VEGFA in HUVECs following hypoxia. HUVECs were exposed to hypoxia and the changes in the expression levels of miR-140-5p and VEGFA were measured using RT-qPCR and western blot analyses. Consistent with the results from the in vivo experiment in rat cerebral tissue, the expression of miR-140-5p was significantly decreased, while the protein expression levels of VEGFA were significantly increased in hypoxic HUVECs compared with those in normoxic HUVECs $(\mathrm{P}<0.01$ and $\mathrm{P}<0.05$, respectively; Fig. 2).

Effect of miR-140-5p on the in vitro angiogenesis of HUVECs following hypoxia. To investigate the effect of miR-140-5p on angiogenesis, the miR-140-5p mimic was transfected into normoxic and hypoxic HUVECs. RT-qPCR analysis demonstrated that, compared with the control group, the expression of miR-140-5p was significantly increased in normoxic and hypoxic HUVECs following transfection ( $\mathrm{P}<0.01$; Fig. $3 \mathrm{~A})$. Subsequently, cell viability, migration and tube formation were examined. An MTT assay demonstrated that cell viability was markedly decreased in normoxic and hypoxic HUVECs following transfection (Fig. 3B). As demonstrated in Fig. 3C, the migrated cell number was significantly different between the control and the miR-140-5p mimic groups $(\mathrm{P}<0.05)$. The results suggested that the enhanced expression of miR-140-5p led to decreased cell migration in the normoxic and hypoxic HUVECs. In addition, compared with the control group, the

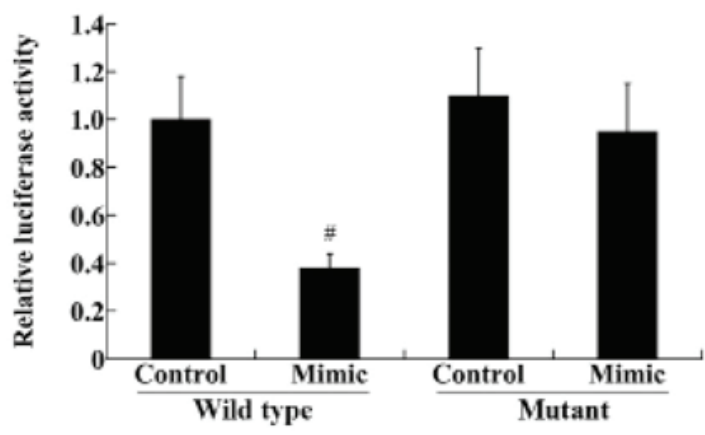

Figure 4. Relative luciferase activity of wild type and the mutant 3 ' untranslated region of vascular endothelial growth factor A in human embryonic kidney 293 cells following transfection with the miR-140-5p mimic. The data are expressed as the mean \pm standard deviation $\left({ }^{\sharp} \mathrm{P}<0.01\right.$, vs. the wild type control group).

tube length was significantly decreased in the normoxic and hypoxic HUVECs following transfection ( $\mathrm{P}<0.05$; Fig. 3D).

miR-140-5p directly targets the 3'UTR of VEGFA and regulates the expression of VEGFA. To reveal the association between miR-140-5p and VEGFA, wild type and mutant 3'UTR of VEGFA were transfected into HEK293 cells, along with the miR-140-5p mimic and miRNA control. The luciferase reporter assay demonstrated that transfection of the miR-140-5p mimic significantly downregulated the luciferase activity in the wild type VEGFA-3'UTR compared with the control group $(\mathrm{P}<0.01$; Fig. 4). However, the mutant VEGFA-3'UTR levels were not significantly altered following transfection (Fig. 4).

Furthermore, the effect of miR-140-5p on the expression of VEGFA in HEK293 cells was examined. As demonstrated in Fig. 5A, the expression of miR-140-5p was significantly upregulated following transfection with the miR-140-5p mimic 
A

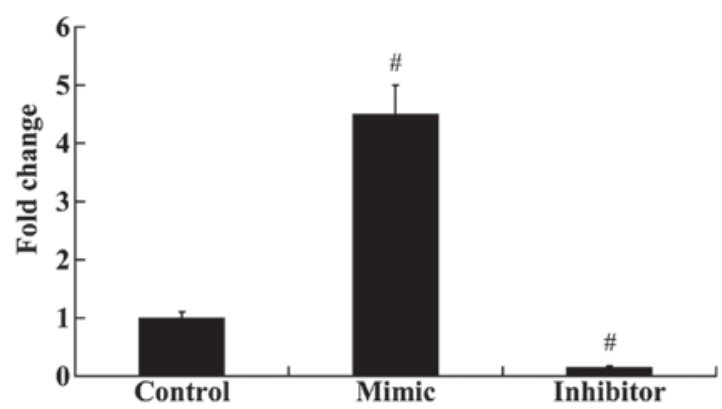

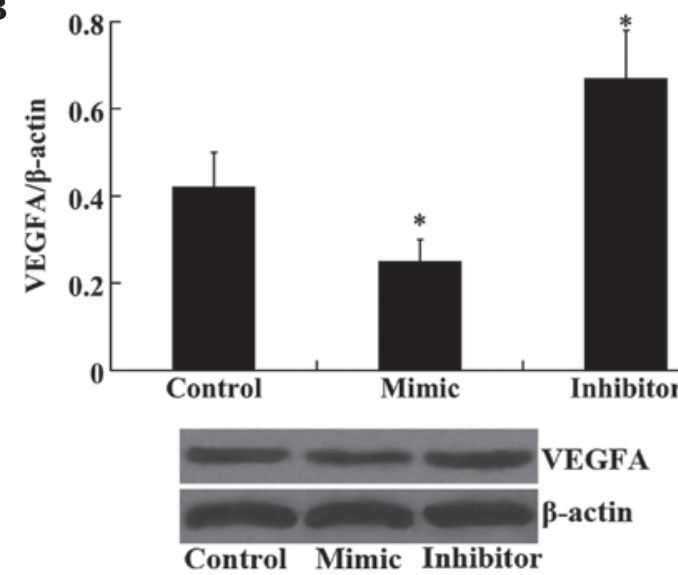

Figure 5. Effect of miR-140-5p on the protein expression of VEGFA in human embryonic kidney 293 cells following transfection with the miR-140-5p mimic and miR-140-5p inhibitor. (A) The fold changes of miR-140-5p was normalized against that of the control group. (B) The protein expression of VEGFA was normalized against $\beta$-actin. The data are expressed as the mean \pm standard deviation $\left({ }^{*} \mathrm{P}<0.05 ;{ }^{*} \mathrm{P}<0.01\right.$, vs. the control group). VEGFA, vascular endothelial growth factor A.

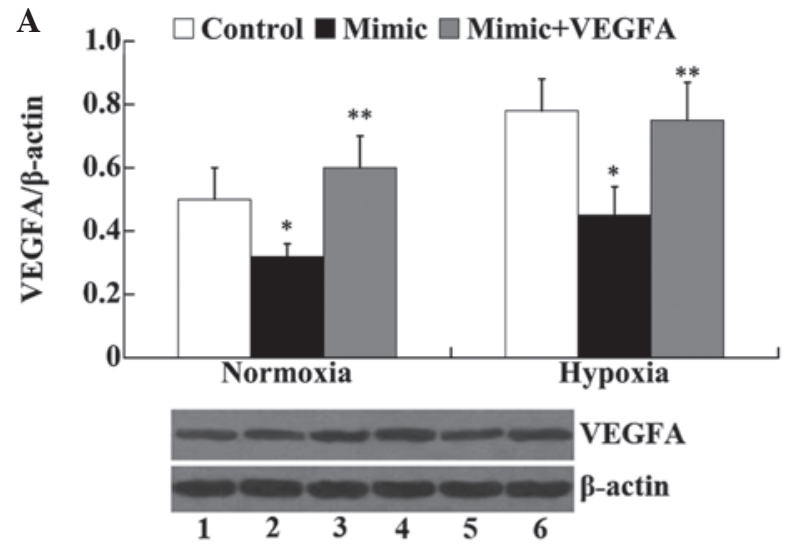

C

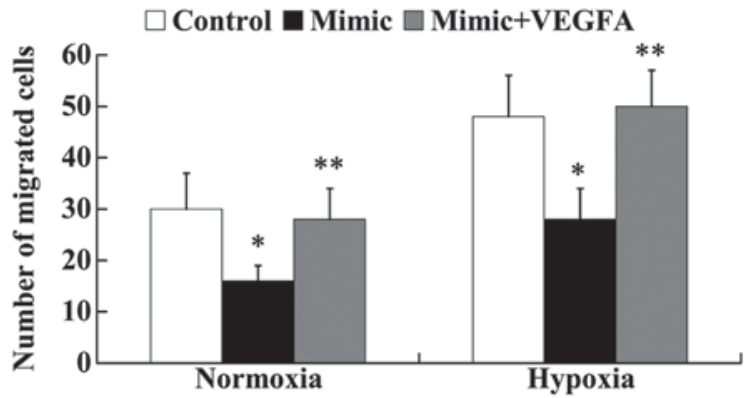

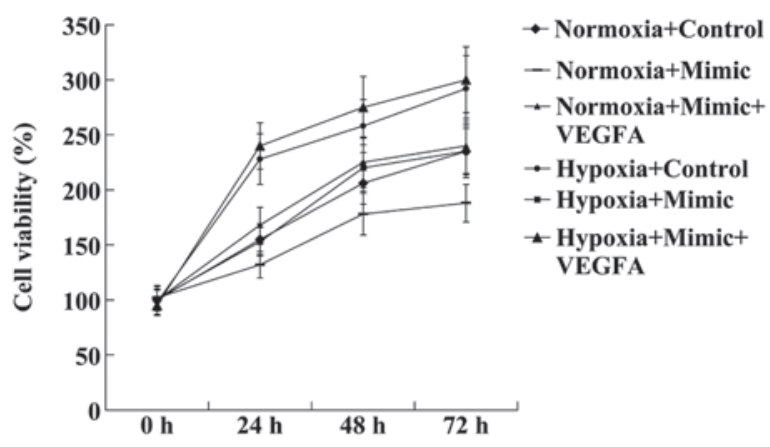

D

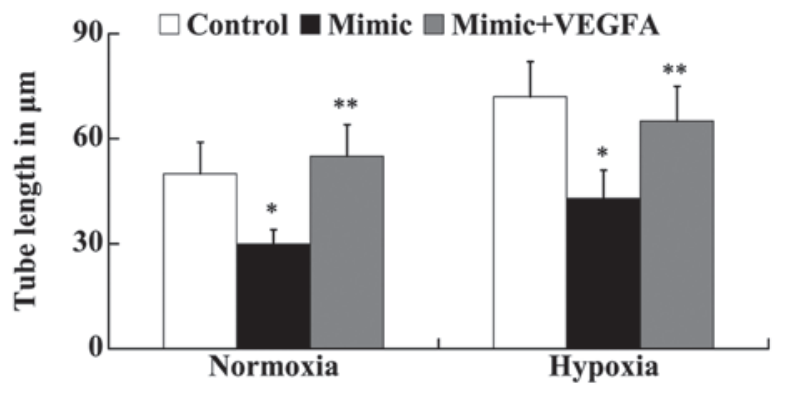

Figure 6. VEGFA attenuates the effect of miR-140-5p on in vitro angiogenesis of human umbilical vein endothelial cells following transfection with the miR-140-5p mimic and VEGFA-pEGFP-C1. (A) The protein expression levels of VEGFA were normalized against $\beta$-actin. (B) Cell viability was determined by 3-(4,5-dimethylthiazol-2-yl)-2,5-diphenyltetrazolium bromide assay. (C) The number of migrated was cells determined using a Transwell migration assay. (D) Tube length was determined using a tube formation assay. The data are expressed as the mean \pm standard deviation ("P<0.05, vs. the control group; ${ }^{* *} \mathrm{P}<0.05$, vs. the mimic group). VEGFA, vascular endothelial growth factor $\mathrm{A}$.

and downregulated following transfection with the miR-140-5p inhibitor, compared with the control group $(\mathrm{P}<0.01)$. Western blot analysis demonstrated that the protein expression levels of VEGFA were significantly decreased following transfection with the miR-140-5p mimic and increased following miR-140-5p inhibitor transfection, compared with the control group $(\mathrm{P}<0.01$; Fig. 5B).

VEGFA attenuates the effect of miR-140-5p on in vitro angiogenesis of HUVECs following hypoxia. To investigate whether the effect of miR-140-5p on angiogenesis was mediated by VEGFA, VEGFA-pEGFP-C1 was transfected into the normoxic and hypoxic HUVECs, together with the miR-140-5p mimic. Western blot analysis demonstrated that in the normoxic and hypoxic HUVECs, miR-140-5p mimic transfection significantly downregulated the protein expression levels of VEGFA compared with the control group $(\mathrm{P}<0.05$; Fig. 6A). However, following mimic + VEGFA transfection, the protein expression levels of VEGFA were significantly increased compared with the mimic group $(\mathrm{P}<0.05$; Fig. 6A). 
An MTT assay demonstrated that the decreased cell viability observed due to the transfection of the miR-140-5p mimic in normoxic and hypoxic HUVECs was reversed by the overexpression of VEGFA (Fig. 6B). The results from the Transwell migration assay revealed that overexpression of VEGFA attenuated the effect of miR-140-5p on cell migration in normoxic and hypoxic HUVECs (Fig. 6C). In addition, compared with the mimic group, the tube length was significantly increased in the mimic + VEGFA group in normoxic and hypoxic HUVECs ( $\mathrm{P}<0.05$; Fig. 6D).

\section{Discussion}

miRNAs are a family of small non-coding RNAs that are 19-24 nucleotides in length. They serve important roles in the regulation of various biological processes, including cell proliferation, differentiation, apoptosis and motility (15-17). Aberrant miRNA expression profiles have been linked to the pathophysiology of numerous human diseases. Previous studies demonstrated that miRNA profiles were altered in tissues, including myocardial, retina, liver and brain tissues, under hypoxic-ischemic conditions (18-21). The miR-140-5p expression profile of rat hippocampus may be detected by microarray (22), and it has been demonstrated that miR-140-5p modulates myelination in the dorsal root ganglion/Schwann cell co-cultures (23). In addition, these previous studies suggested that miR-140-5p may be a functional miRNA of the central nervous system. In the present study, it was demonstrated that the expression of miR-140-5p was significantly decreased in rat cerebral ischemia and in the in vitro hypoxic condition. These findings suggested that miR-140-5p may be involved in the pathogenesis of hypoxic-ischemic brain damage.

Ischemic stroke results from the blockage of a brain arterial blood vessel, therefore, the improvement of blood supply in the ischemic brain tissue is the fundamental approach for the treatment of ischemic stroke. Previous clinical studies indicated that good collateral circulation may influence the infarct volume and aid in the prognosis of patients with ischemic stroke (24-27). Cerebral collateral circulation is a secondary compensatory mechanism following cerebral ischemia, and it refers to the collateral or the newly formed vascular anastomosis that stabilize cerebral blood flow when the arteries are blocked.

Angiogenesis is the formation of new vessels sprouting from pre-existing capillaries (28), and it was suggested to be a potent therapy for ischemic stroke through increasing the cerebral blood flow (29). A previous study indicated that angiogenesis is associated with the neurological functional recovery and has a beneficial effect on the long-term survival of patients following cerebral ischemia (30).

Cell proliferation, migration and tube formation in endothelial cells are important processes in angiogenesis. Previous studies in cancer research indicated that miR-140-5p suppresses cancer cell proliferation and metastasis $(9,11)$. In the present study, an in vitro study was performed for the first time to the best of our knowledge, to investigate the effect of miR-140-5p on angiogenesis in normoxic and hypoxic HUVECs. The results demonstrated that miR-140-5p exerts inhibitory effects against angiogenesis in normoxic and hypoxic HUVECs, as indicated by decreased cell proliferation, migration and tube formation in HUVECs following transfection with the miR-140-5p mimic. These results suggested that miR-140-5p may regulate angiogenesis following hypoxic-ischemic brain damage.

VEGFA is a notable pro-angiogenic factor, which promotes angiogenesis through numerous mechanisms (31). It has been demonstrated that VEGFA may induce the proliferation and migration of endothelial cells, and increase vascular permeability (32). VEGFA has important roles in the angiogenesis following cerebral ischemia (33-35). Several animal and clinical studies have demonstrated that VEGFA is significantly upregulated following stroke $(36,37)$. Administration of VEGFA appears to increase microvessel density in the ischemic penumbra following MCAO in rats, thus leading to an enhanced post-ischemic angiogenesis (38). To investigate the association between miR-140-5p and VEGFA, potential targets of miR-140-5p were identified using the miRanda algorithm (www.microrna.org) and VEGFA was predicted to be a direct target. The luciferase reporter assay demonstrated that miR-140-5p may directly target the 3'UTR of VEGFA and downregulated the protein expression of VEGFA. Furthermore, overexpression of VEGFA may attenuate the effect of miR-140-5p on angiogenesis.

In conclusion, the results of the present study demonstrated that miR-140-5p suppresses angiogenesis following cerebral ischemia, and this effect is achieved by directly targeting VEGFA. The present study provided evidence that miR-140-5p may be a potential candidate for the treatment of ischemic brain injury.

\section{References}

1. Wang X: Investigational anti-inflammatory agents for the treatment of ischaemic brain injury. Expert Opin Investig Drugs 14: 393-409, 2005.

2. Durukan A and Tatlisumak T: Acute ischemic stroke: Overview of major experimental rodent models, pathophysiology, and therapy of focal cerebral ischemia. Pharmacol Biochem Behav 87: 179-197, 2007.

3. Arenillas JF, Sobrino T, Castillo J and Dávalos A: The role of angiogenesis in damage and recovery from ischemic stroke. Curr Treat Options Cardiovasc Med 9: 205-212, 2007.

4. Navarro-Sobrino M, Rosell A, Hernández-Guillamon M, Penalba A, Boada C, Domingues-Montanari S, Ribó M, Alvarez-Sabín J and Montaner J: A large screening of angiogenesis biomarkers and their association with neurological outcome after ischemic stroke. Atherosclerosis 216: 205-211, 2011.

5. Koutsis G, Siasos G and Spengos K: The emerging role of microRNA in stroke. Curr Top Med Chem 13: 1573-1588, 2013.

6. Peng T, Jia YJ, Wen QQ, Guan WJ, Zhao EY and Zhang BA: Expression of microRNA in neonatal rats with hypoxic-ischemic brain damage. Chin J Contemp Pediatr 12: 373-376, 2012 (In Chinese).

7. Hatse S, Brouwers B, Dalmasso B, Laenen A, Kenis C, Schöffski P and Wildiers H: Circulating MicroRNAs as easy-to-measure aging biomarkers in older breast cancer patients: Correlation with chronological age but not with fitness/frailty status. PLoS One 9: e110644, 2014.

8. Mosakhani N, Lahti L, Borze I, Karjalainen-Lindsberg ML, Sundström J, Ristamäki R, Osterlund $\mathrm{P}$, Knuutila S and Sarhadi VK: MicroRNA profiling predicts survival in anti-EGFR treated chemorefractory metastatic colorectal cancer patients with wild-type KRAS and BRAF. Cancer Genet 205: 545-551, 2012.

9. Yang H, Fang F, Chang R and Yang L: MicroRNA-140-5p suppresses tumor growth and metastasis by targeting transforming growth factor $\beta$ receptor 1 and fibroblast growth factor 9 in hepatocellular carcinoma. Hepatology 58: 205-217, 2013. 
10. LiW andHeF: Monocyte tomacrophage differentiation-associated (MMD) targeted by miR-140-5p regulates tumor growth in non-small cell lung cancer. Biochem Biophys Res Commun 450: 844-850, 2014

11. Kai Y, Peng W, Ling W, Jiebing h and Zhuan B: Reciprocal effects between microRNA-140-5p and ADAM10 suppress migration and invasion of human tongue cancer cells. Biochem Biophys Res Commun 448: 308-314, 2014.

12. National Research Council: Guide for the Care and Use of Laboratory Animals. $8^{\text {th }}$ edition. National Academies Press, 2011.

13. Longa EZ, Weinstein PR, Carlson S and Cummins R: Reversible middle cerebral artery occlusion without craniectomy in rats. Stroke 20: 84-91, 1989.

14. Livak KJ and Schmittgen TD: Analysis of relative gene expression data using real-time quantitative PCR and the 2(-Delta Delta C(T)) Method. Methods 25: 402-408, 2001.

15. Cheng AM, Byrom MW, Shelton J and Ford LP: Antisense inhibition of human miRNAs and indications for an involvement of miRNA in cell growth and apoptosis. Nucleic Acids Res 33: 1290-1297, 2005.

16. Wen KC, Sung PL, Yen MS, Chuang CM, Liou WS and Wang PH MicroRNAs regulate several functions of normal tissues and malignancies. Taiwan J Obstet Gynecol 52: 465-469, 2013.

17. Chan SH and Wang LH: Regulation of cancer metastasis by microRNAs. J Biomed Sci 22: 9, 2015.

18. Roy S, Khanna S, Hussain SR, Biswas S, Azad A, Rink C, Gnyawali S, Shilo S, Nuovo GJ and Sen CK: MicroRNA expression in response to murine myocardial infarction: miR-21 regulates fibroblast metalloprotease-2 via phosphatase and tensin homologue. Cardiovasc Res 82: 21-29, 2009.

19. Shen J, Yang X, Xie B, Chen Y, Swaim M, Hackett SF and Campochiaro PA: MicroRNAs regulate ocular neovascularization. Mol Ther 16: 1208-1216, 2008.

20. Dharap A, Bowen K, Place R, Li LC and Vemuganti R: Transient focal ischemia induces extensive temporal changes in rat cerebral microRNAome. J Cereb Blood Flow Metab 29: 675-687, 2009.

21. $\mathrm{Xu} \mathrm{CF}, \mathrm{Yu} \mathrm{CH}$ and Li YM: Regulation of hepatic microRNA expression in response to ischemic preconditioning following ischemia/reperfusion injury in mice. OMICS 13: 513-520, 2009.

22. Park CS and Tang SJ: Regulation of microRNA expression by induction of bidirectional synaptic plasticity. J Mol Neurosci 38 50-56, 2009.

23. Viader A, Chang LW, Fahrner T, Nagarajan R and Milbrandt J: MicroRNAs modulate Schwann cell response to nerve injury by reinforcing transcriptional silencing of dedifferentiation-related genes. J Neurosci 31: 17358-17369, 2011.

24. Miteff F, Levi CR, Bateman GA, Spratt N, McElduff P and Parsons MW: The independent predictive utility of computed tomography angiographic collateral status in acute ischaemic stroke. Brain 132: 2231-2238, 2009.

25. Liebeskind DS, Cotsonis GA, Saver JL, Lynn MJ, Turan TN, Cloft HJ and Chimowitz MI; Warfarin-Aspirin Symptomatic Intracranial Disease (WASID) Investigators: Collaterals dramatically alter stroke risk in intracranial atherosclerosis. Ann Neurol 69: 963-974, 2011.
26. Rusanen H, Saarinen JT and Sillanpää N: Collateral Circulation Predicts the Size of the Infarct Core and the Proportion of Salvageable Penumbra in Hyperacute Ischemic Stroke Patients Treated with Intravenous Thrombolysis. Cerebrovasc Dis 40: 182-190, 2015.

27. Ernst M, Forkert ND, Brehmer L, Thomalla G, Siemonsen S, Fiehler $J$ and Kemmling A: Prediction of infarction and reperfusion in stroke by flow- and volume-weighted collateral signal in MR angiography. AJNR Am J Neuroradiol 36: $275-282,2015$.

28. Folkman J and Shing Y: Angiogenesis. J Biol Chem 267: 10931-10934, 1992.

29. Henry TD: Therapeutic angiogenesis. BMJ 318: 1536-1539, 1999.

30. Beck H and Plate KH: Angiogenesis after cerebral ischemia. Acta Neuropathol 117: 481-496, 2009.

31. Matsui M and Tabata Y: Enhanced angiogenesis by multiple release of platelet-rich plasma contents and basic fibroblast growth factor from gelatin hydrogels. Acta Biomater 8: 1792-1801, 2012

32. Spyridopoulos I, Luedemann C, Chen D, Kearney M, Chen D, Murohara T, Principe N, Isner JM and Losordo DW: Divergence of angiogenic and vascular permeability signaling by VEGF: Inhibition of protein kinase C suppresses VEGF-induced angiogenesis, but promotes VEGF-induced, NO-dependent vascular permeability. Arterioscler Thromb Vasc Biol 22: 901-906, 2002.

33. Plate KH, Beck H, Danner S, Allegrini PR and Wiessner C: Cell type specific upregulation of vascular endothelial growth factor in an MCA-occlusion model of cerebral infarct. J Neuropathol Exp Neurol 58: 654-666, 1999.

34. Banai S, Jaklitsch MT, Shou M, Lazarous DF, Scheinowitz M, Biro S, Epstein SE and Unger EF: Angiogenic-induced enhancement of collateral blood flow to ischemic myocardium by vascular endothelial growth factor in dogs. Circulation 89: 2183-2189, 1994.

35. Lennmyr F, Ata KA, Funa K, Olsson Y and Terént A: Expression of vascular endothelial growth factor (VEGF) and its receptors (Flt-1 and Flk-1) following permanent and transient occlusion of the middle cerebral artery in the rat. J Neuropathol Exp Neurol 57: 874-882, 1998.

36. Marti HJ, Bernaudin M, Bellail A, Schoch H, Euler M, Petit E and Risau W: Hypoxia-induced vascular endothelial growth factor expression precedes neovascularization after cerebral ischemia. Am J Pathol 156: 965-976, 2000.

37. Hayashi T, Abe K, Suzuki H and Itoyama Y: Rapid induction of vascular endothelial growth factor gene expression after transient middle cerebral artery occlusion in rats. Stroke 28: 2039-2044, 1997.

38. Zhang ZG, Zhang L, Jiang Q, Zhang R, Davies K, Powers C, Bruggen $\mathrm{N}$ and Chopp M: VEGF enhances angiogenesis and promotes blood-brain barrier leakage in the ischemic brain. J Clin Invest 106: 829-838, 2000. 